\title{
A COERÊNCIA NA COPRODUÇÃO DE UM MODELO DE CONHECIMENTO EM UM PROJETO DE PESQUISA INTERDISCIPLINAR
}

\author{
Viviane Schneider ${ }^{1}$; \\ Denilson Sell'; \\ Aran Bey Tcholakian Morales ${ }^{3}$; \\ Jose Leomar Todesco ${ }^{4}$; \\ Roberto Carlos Dos Santos Pacheco ${ }^{5}$
}

\begin{abstract}
Coherence in co-production between agents is a determining factor in the quality of the resulting research artifacts. Such coherence can be assessed to verify the quality of research and studies produced, as well as to direct actions for improvement and interaction between the agents that co-produce. This case study describes an analysis of coherence in the development of a knowledge model. This analysis was carried out in four dimensions, namely: (i) the researchers' sense of unity, (ii) the symbolic referentiality, (iii) the receptivity of the norms and conceptual topics that permeate the project, as well as, (iv) the prescriptivity, which represents the alignment between planned and executed. The results of this case study helped to improve the co-production activities of a research and development project.
\end{abstract}

Keywords: Coherence; Coproduction; Knowledge model; Resilience; Human Factors.

Resumo: A coerência na coprodução entre agentes é um fator determinante na qualidade dos artefatos de pesquisas resultantes. Tal coerência pode ser avaliada para verificar a qualidade de pesquisas e estudos produzidos, bem como, para direcionar ações de melhoria e interação entre os agentes que coproduzem. Neste estudo de caso é descrito uma análise da coerência no desenvolvimento de um modelo de conhecimento. Esta análise foi realizada em quatro dimensões, sendo elas: (i) o senso de unidade dos pesquisadores; (ii) a referencialidade simbólica; (iii) a receptividade das normas e tópicos conceituais que permeiam o projeto; e (iv) a prescritividade, que representa o alinhamento entre o planejado e o executado. Os resultados

\footnotetext{
${ }^{1}$ Engenharia e Gestão do Conhecimento - Universidade Federal de Santa Catarina (UFSC) Florianópolis - Brasil. Correio eletrônico: viviane.sch@gmail.com

2 Engenharia e Gestão do Conhecimento - Universidade Federal de Santa Catarina (UFSC) e Universidade do Estado de Santa Catarina (UDESC) Florianópolis - Brasil. Correio eletrônico: denilson@stela.org.br

${ }^{3}$ Engenharia e Gestão do Conhecimento - Universidade Federal de Santa Catarina (UFSC) Florianópolis - Brasil. Correio eletrônico: aran@stela.org.br

${ }^{4}$ Engenharia e Gestão do Conhecimento - Universidade Federal de Santa Catarina (UFSC) Florianópolis Brasil. Correio eletrônico: titetodesco@gmail.com

${ }^{5}$ Engenharia e Gestão do Conhecimento - Universidade Federal de Santa Catarina (UFSC) Florianópolis Brasil. Correio eletrônico: rpacheco@egc.ufsc.br
}

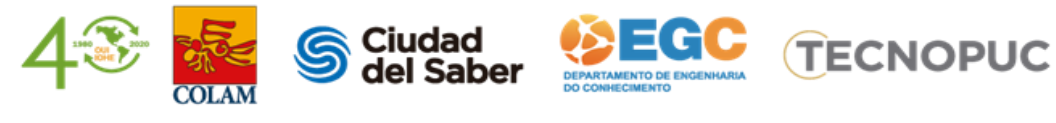


deste estudo de caso auxiliou na melhorias das atividades de coprodução de um projeto de pesquisa e desenvolvimento.

Palavras-chave: Coerência; Coprodução; Modelo de conhecimento; Resiliência; Fatores Humanos.

\section{INTRODUÇÃO}

O presente artigo aborda a análise da coerência na coprodução de conhecimento no âmbito de um projeto de pesquisa interdisciplinar que mobilizou diversas empresas do setor de óleo e gás além de universidades e pesquisadores independentes. O projeto busca estabelecer uma abordagem interdisciplinar para a identificação de estratégias para o fortalecimento da resiliência em operações integradas no setor de óleo e gás, envolvendo as áreas de Engenharia de Resiliência (ER), Engenharia e Gestão do Conhecimento (EGC), Serviço Social (SS), Sociologia (SO) e Meio Ambiente (MA).

Neste projeto, um dos elementos que mobilizou a participação de diferentes atores foi a produção de um sistema de indicadores, englobando variáveis que buscam descrever/mensurar os fatores que condicionam respostas resilientes e impactar a segurança de operações no setor de óleo e gás. Para viabilizar a aplicação do sistema em uma prova de conceito, a pesquisa culminou na criação de um modelo conceitual que reúne os diferentes fatores que condicionam o desempenho resiliente. Para tanto, foi estabelecido um construto de segunda ordem denominado Performance Conditionings descrito em um modelo de conhecimento ${ }^{6}$ contendo três constructos de primeira ordem: (i) indivíduo, (ii) trabalho e (iii) organização. Esses três constructos representam as interfaces do modelo SHELL (Software, Hardware, Environment, Liveware-Liveware) (Hawkins; Orlady, 1993).

O modelo de conhecimento foi desenvolvido no projeto para permitir a integração das diversas análises realizadas pelos grupos de pesquisa e possibilitar uma ampla e aprofundada análise sobre os fatores humanos em operações integradas. Conforme ilustra a Figura 1, os três constructos classificam 10 (dez) fatores, os quais, por sua vez, foram detalhados em 148 fatores

\footnotetext{
${ }^{6}$ Adota-se neste projeto a definição de Schreiber et. al. (2012), no qual um modelo de conhecimento objetiva explicar em detalhes os tipos e estruturas do conhecimento usado na execução de uma tarefa.
} 
associados, organizados em um modelo hierárquico com três níveis. $\mathrm{O}$ objetivo desse modelo é apresentar um agregador interdisciplinar das diversas facetas de coleta, análises de dados e informações sobre fatores humanos e resiliência, com vistas a facilitar o desenvolvimento de um índice capaz de representar o nível de desenvolvimento dos fatores que condicionam o desempenho resiliente em operações integradas. Na Figura 1, estão dispostos os principais fatores organizados no modelo de conhecimento, o qual teve como base principal as perspectivas disciplinares dos pesquisadores sobre fatores humanos e sua relação com a resiliência em operações complexas e com alto nível de risco. Para a estruturação do modelo foram analisados ainda modelos aplicados em diferentes indústrias, tais como os descritos em European Railway Agency (2013), ANSI/ASIS (2009), Petit et al. 2013 e ISO 22316 (2017).

Figura 1 - Visão de geral dos Construtos e Fatores da Dimensão Performance Conditionings

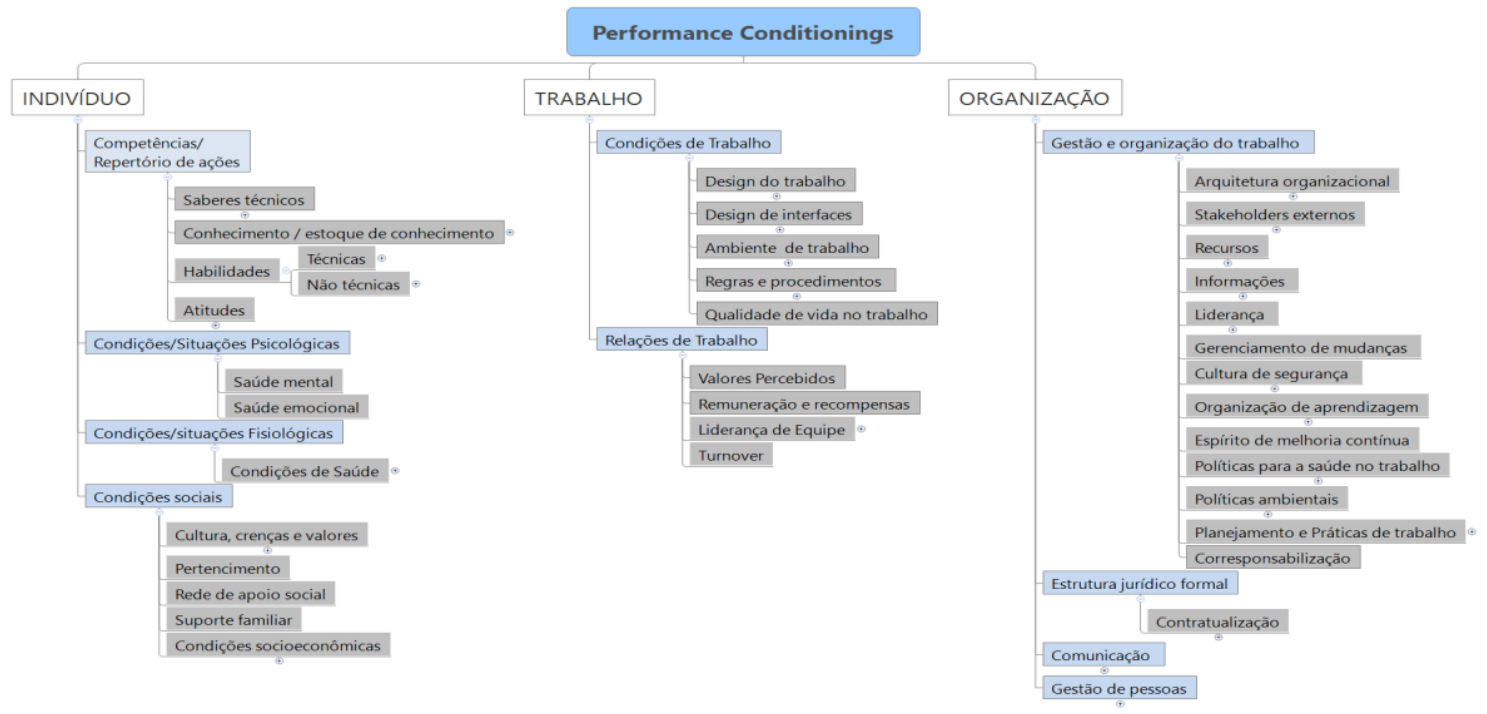

Fonte: relatório de pesquisa do projeto interdisciplinar.

O constructo Indivíduo reúne quatro fatores, a saber: a) Competências/Repertório de ações; b) Condições/Situações Psicológicas; c) Condições/Situação Fisiológicas e d) Condições Sociais. Os quatro fatores reúnem 72 fatores associados, organizados em até três níveis, sendo 13 fatores associados no nível um, 24 fatores associados no nível dois, e 33 fatores associados no nível três. O constructo Trabalho possui dois fatores: a) Condições de trabalho e b) Relações de Trabalho. Os dois fatores reúnem 9 fatores associados no nível um, 29 fatores associados no nível dois, e 27 fatores associados no nível três. O constructo Organização possui quatro fatores, a saber: a) Gestão e Organização do trabalho, b) Estrutura Formal e Jurídica, c) Comunicação,

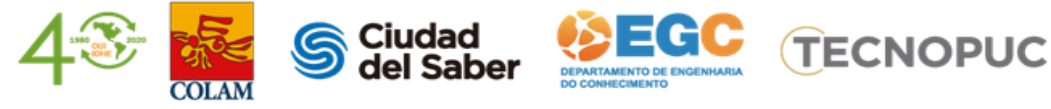


e d) Gestão de Pessoas. Os quatro fatores reúnem 26 fatores associados de nível um, 26 fatores associados de nível dois e 5 fatores associados de nível três. Os dados coletados pelas equipes integrantes do projeto serviram de insumo para alimentação do modelo e para orientar o cálculo de indicadores. $\mathrm{O}$ algoritmo de cálculo está incorporado na prova de conceito de sistema de conhecimento que possibilita a apresentação dos indicadores e análises qualitativas. O modelo faz parte de um framework no qual o modelo atua como norteador da coleta de dados nas empresas envolvidas no estudo, bem como base da interface do ferramental de apoio a gestão da segurança nas plataformas.

A coprodução do modelo ocorreu durante dois anos de projeto, e teve a participação de mais de 30 pesquisadores, entre mestres e doutores, além de especialistas da indústria de óleo e gás. Esses atores participaram de oficinas para identificar e discutir os fatores que deveriam compor o modelo de análise. Oficinas foram realizadas também para a autoavaliação dos trabalhos de coprodução realizados, no que tange ao desenvolvimento do modelo de conhecimento do projeto. O objetivo dessa autoavaliação foi verificar a coerência no contexto da coprodução do modelo de conhecimento do projeto, com vistas a verificar oportunidades de melhoria no processo de coprodução em projetos futuros, bem como avaliar potenciais benefícios de ampliação da verificação de coerência da coprodução entre as equipes mobilizadas nas operações das empresas envolvidas no estudo.

Para avaliar as características das ações de coprodução realizadas neste projeto, o escopo de aplicação seguiu as instruções de implementação do método proposto em Schneider (2019). A coprodução avaliada encontra-se nas interações ocorridas para desenvolver o modelo de conhecimento do projeto. A partir dos conteúdos de apoio ao estabelecimento do modelo, foram identificadas as fontes de dados para compor a avaliação. Essas fontes são provenientes de análises documentais e percepção dos membros da equipe de pesquisa.

\section{REFERENCIAL TEÓRICO}

O método de avaliação foi desenvolvido a partir dos estudos de Schneider (2019), tese que apresenta um metamodelo para avaliação da coerência de símbolos que unem agentes em contextos sociotécnicos de coprodução. A auto avaliação partiu de um metamodelo teórico da 
tese de Schneider (2019), o qual visa mapear os símbolos que unem agentes em contextos sociotécnicos de coprodução. O intuito dessa aplicação foi realizar um piloto com o metamodelo, visando identificar os benefícios de aplicação em fases futuras do projeto de pesquisa. O metamodelo teórico é apresentado na Figura 2 e detalhado em Schneider (2019).

Figura 2 - Metamodelo teórico de Schneider (2019)

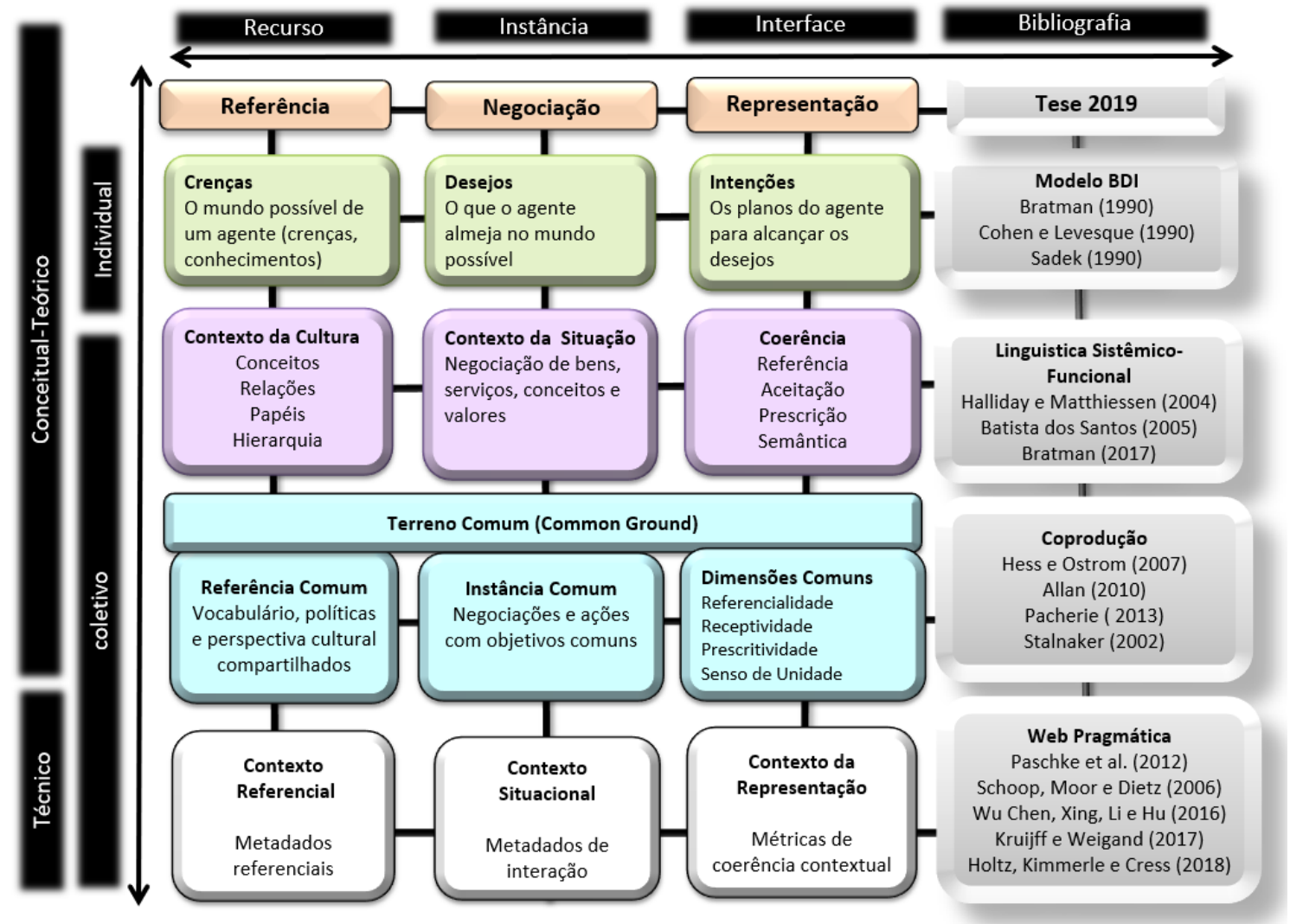

Fonte: Schneider (2019).

Conforme Schneider (2019), o Contexto Referencial representa uma estrutura de dados sobre regras, sanções, objetivos, metas e resultados esperados em uma rede de coprodução, bem como, os conceitos basilares, membros, papéis e relevantes para a rede. Tais dados são criados a partir das negociações iniciais dos processos coprodutivos. Neste projeto foram feitas avaliações sobre os contratos e documentos reunidos pelas empresas e pesquisadores participantes do projeto de pesquisa. O Contexto Situacional representa as ações instanciadas do contexto referencial, mas que possuem aspectos adaptativos ao contexto local em que ocorrem. As características tratadas nesse contexto envolvem as comunicações entre agentes (humanos ou não), as negociações de valores, a coprodução constante de evoluções de 
conceitos, bens, serviços e informações. Esse contexto é flexível, pois se adapta ao dinamismo de uma dada situação interativa. Nesse contexto, ocorrem as adaptações de regras gerais, que são interpretadas para atender a aspectos locais de coprodução. O Contexto da Representação refere-se às representações que sintetizam o status atual da rede de coprodução quanto à coerência e apresenta as dimensões e os indicadores da coprodução mais relevantes para a comunidade, em termos de coerência entre os contextos. Conforme ilustra a Figura 3 a análise da coerência contextual é realizada por meio de quatro dimensões: Prescritividade, Referencialidade, Senso de Unidade, e Receptividade.

Figura 3 - Metamodelo Conceitual de Schneider (2019)

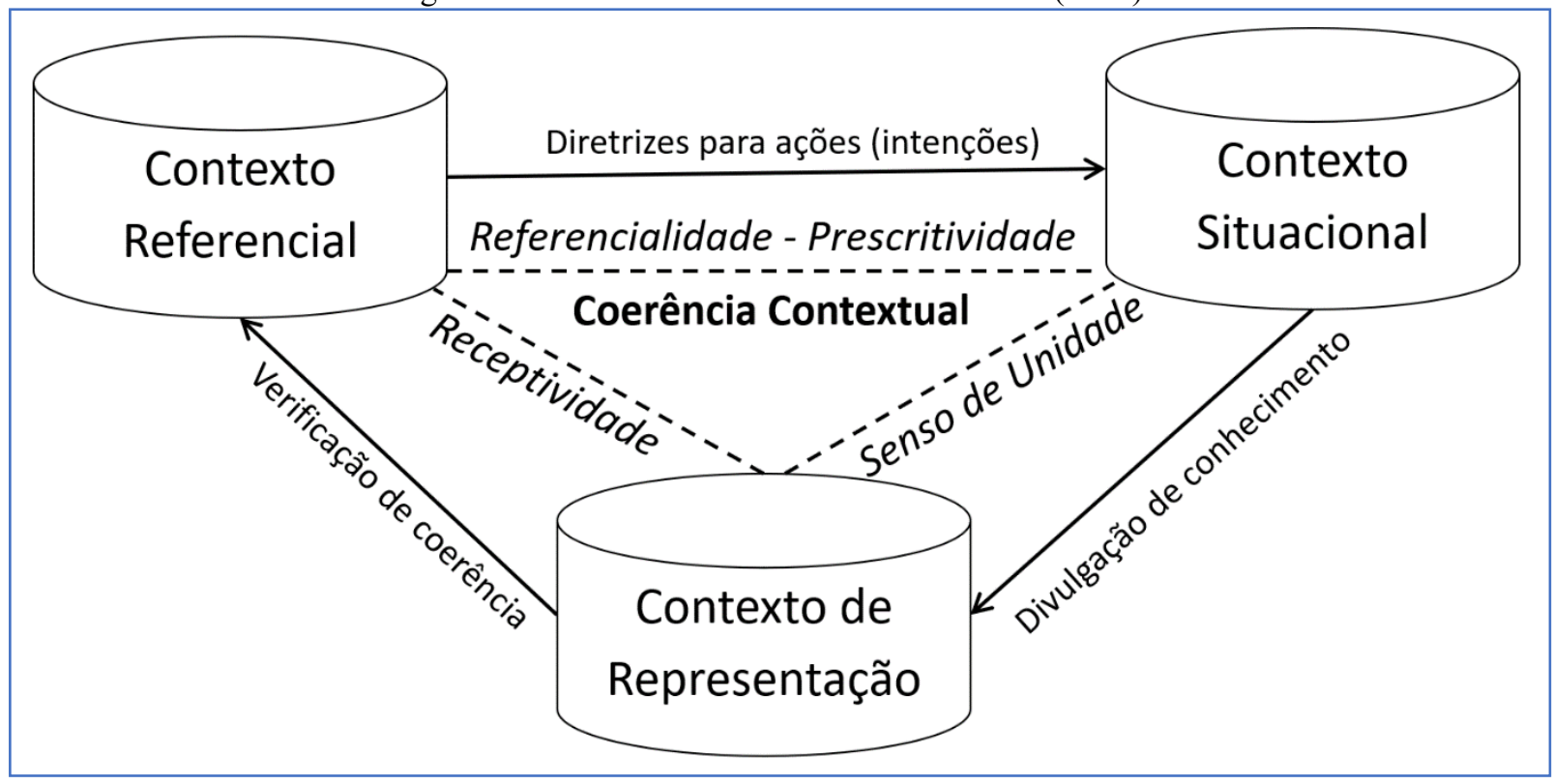

Fonte: Schneider (2019).

A próxima seção apresenta os dados produzidos na aplicação do metamodelo de Schneider (2019).

\section{DADOS DO CONTEXTO REFERENCIAL NO ÂMBITO DO PROJETO}

O Conjunto de dados Referential_Common_Contract, apresentado no Quadro 1, descreve as análises qualitativas referentes aos elementos que simbolizam o contrato comum referencial da coprodução entre as atividades desenvolvidas pelos integrantes de um dos grupos do projeto (identificado por Grupo A) e das atividades desenvolvidas pelo Grupo A com os 
demais grupos deste projeto, conforme orientações e adaptações do metamodelo de Schneider (2019). Os valores descritos foram estabelecidos por meio de análises documentais e entrevistas com os integrantes do Grupo A integrante do projeto de pesquisa interdisciplinar.

\begin{tabular}{|c|c|c|c|c|c|}
\hline Simb. & Componentes & Observações & Valor & Peso & Total \\
\hline CO1 & $\begin{array}{l}\text { Resultado } \\
\text { comum }\end{array}$ & $\begin{array}{l}\text { Modelo de conhecimento para o framework } \\
\text { de avaliação de resiliência }\end{array}$ & 1 & 0,33333333 & 0,33333333 \\
\hline $\mathrm{CO} 2$ & Propósito de uso & $\begin{array}{l}\text { Avaliação dos fatores que condicionam as } \\
\text { respostas resilientes em operações } \\
\text { integradas. Essa finalidade não foi } \\
\text { estabelecida no referencial, foi construída ao } \\
\text { longo da coprodução }\end{array}$ & 0,5 & 0,33333333 & 0,16666667 \\
\hline $\mathrm{CO} 3$ & Precificação & $\begin{array}{l}\text { O preço foi estabelecido no contrato do } \\
\text { projeto }\end{array}$ & 1 & 0,33333333 & 0,33333333 \\
\hline QtO & $\begin{array}{l}\text { Qualidade do } \\
\text { resultado comum }\end{array}$ & $\begin{array}{l}\text { As fases e etapas foram sendo adaptadas ao } \\
\text { longo do projeto. Elas foram estabelecidas } \\
\text { parcialmente com os demais grupos de } \\
\text { pesquisa. }\end{array}$ & 0,5 & 1 & 0,5 \\
\hline Q1O & $\begin{array}{l}\text { Quantidade do } \\
\text { resultado comum }\end{array}$ & $\begin{array}{l}\text { Os pacotes de trabalho foram definidos no } \\
\text { contrato. Algumas modificações foram } \\
\text { ocorrendo ao longo do projeto }\end{array}$ & 0,8 & 1 & 0,8 \\
\hline DCO & Meta de tempo & $\begin{array}{l}\text { Todas as etapas de entregáveis foram } \\
\text { previamente acordados }\end{array}$ & 1 & 1 & 1 \\
\hline CG & Objetivo Comum & $\begin{array}{l}\text { O objetivo comum foi estabelecido dentro } \\
\text { do Grupo A, mas a definição do objetivo } \\
\text { comum com os demais grupos foi difícil de } \\
\text { ser estabelecida }\end{array}$ & 0,7 & 1 & 0,7 \\
\hline GCA1 & $\begin{array}{l}\text { Guia de } \\
\text { coordenação de } \\
\text { ações coletivas }\end{array}$ & $\begin{array}{l}\text { O modelo de atuação com os demais grupos } \\
\text { foi estabelecido ao longo do projeto, } \\
\text { seguindo um processo de aproximação } \\
\text { gradual entre as frentes do projeto }\end{array}$ & 0,3 & 0,2 & 0,06 \\
\hline GCA2 & Coordenação & $\begin{array}{l}\text { Houve a coordenação via e-mail para o } \\
\text { agendamento de reuniões. Os espaços de } \\
\text { interação entre os grupos foram significados } \\
\text { ao longo do projeto }\end{array}$ & 0,5 & 0,2 & 0,1 \\
\hline GCA3 & Repositório & $\begin{array}{l}\text { Um repositório de documentos foi } \\
\text { estabelecido, mas tal repositório enfatizou a } \\
\text { organização do conjunto de artefatos finais } \\
\text { das entregas do projeto. Parte do conteúdo } \\
\text { produzido ao longo da pesquisa ficou } \\
\text { restrito aos grupos de maneira } \\
\text { individualizada }\end{array}$ & 0,7 & 0,2 & 0,14 \\
\hline GCA4 & Interação & $\begin{array}{l}\text { Ferramentas de videoconferência com } \\
\text { Whereby, Zoom e Skype foram utilizados }\end{array}$ & 1 & 0,2 & 0,2 \\
\hline GCA5 & Reuniões & $\begin{array}{l}\text { No Grupo A havia reuniões semanais. } \\
\text { Reuniões envolvendo todas as equipes eram } \\
\text { esporádicas }\end{array}$ & 1 & 0,2 & 0,2 \\
\hline GIA & $\begin{array}{l}\text { Guia de ação } \\
\text { individual }\end{array}$ & $\begin{array}{l}\text { Há um registro das ações individuais, } \\
\text { disponíveis em Excel, porém essas } \\
\text { descrições são parciais, pois o método de } \\
\text { como as ações se interrelacional não foi } \\
\text { definido }\end{array}$ & 0,4 & 1 & 0,4 \\
\hline GCV & $\begin{array}{l}\text { Guia de } \\
\text { coordenação de } \\
\text { ações voluntárias }\end{array}$ & $\begin{array}{l}\text { Não formalizado, as ações foram } \\
\text { estabelecidas de maneira espontânea ao } \\
\text { longo do projeto }\end{array}$ & 0 & 1 & 0 \\
\hline
\end{tabular}

\section{4}




\begin{tabular}{|c|c|c|c|c|c|}
\hline Simb. & Componentes & Observações & Valor & Peso & Total \\
\hline IO & $\begin{array}{l}\text { Propriedade } \\
\text { Intelectual }\end{array}$ & $\begin{array}{l}\text { Foi estabelecido um contrato de propriedade } \\
\text { intelectual, mas dúvidas restaram sobre a } \\
\text { possibilidade de menção ao estudo em parte } \\
\text { do projeto }\end{array}$ & 0,5 & 1 & 0,5 \\
\hline $\mathrm{CR}$ & $\begin{array}{l}\text { Regras de } \\
\text { Consenso } \\
\end{array}$ & $\begin{array}{l}\text { Não estava claro no contrato, mas na leitura } \\
\text { fica parcialmente implícito }\end{array}$ & 0,5 & 1 & 0,5 \\
\hline FVO & $\begin{array}{l}\text { Objetos de valor } \\
\text { de primeira } \\
\text { ordem }\end{array}$ & $\begin{array}{l}\text { Pacotes de trabalho descritos no contrato } \\
\text { foram sendo significados e modificados ao } \\
\text { longo do projeto. }\end{array}$ & 0,8 & 1 & 0,8 \\
\hline SVO & $\begin{array}{l}\text { Objetos de valor } \\
\text { de segunda } \\
\text { ordem }\end{array}$ & $\begin{array}{l}\text { Não estava descrito no contrato. Foi } \\
\text { estabelecido parcialmente ao longo do } \\
\text { projeto }\end{array}$ & 0,5 & 1 & 0,5 \\
\hline HRcc & Código Hash & $\begin{array}{l}\text { Possui um contrato firmado, equivalente ao } \\
\text { código Hash }\end{array}$ & 1 & 1 & 1 \\
\hline
\end{tabular}

Fonte: elaborado pelos autores.

O Conjunto de dados Reference_Cultural_Perspective, apresentado no Quadro 2, descreve as análises qualitativas referentes aos elementos que simbolizam a cultura da coprodução do Grupo A, em relação aos demais grupos deste projeto, conforme orientações e adaptações do metamodelo de Schneider (2019). Os valores descritos foram estabelecidos por meio de análises documentais e entrevistas com os integrantes do Grupo A.

Quadro 2- Conjunto de dados Reference_Cultural_Perspective (Perspectiva cultural de referência)

\begin{tabular}{|c|c|c|c|c|c|}
\hline Simb. & Componentes & Observações & Valor & Peso & Total \\
\hline FAZ1 & $\begin{array}{l}\text { Conjunto de } \\
\text { atributos de campo }\end{array}$ & $\begin{array}{l}\text { O processo de definição dos atributos do } \\
\text { modelo envolveu todos os integrantes do } \\
\text { projeto, mas a revisão de parte dos } \\
\text { elementos não foi possível em virtude da } \\
\text { dificuldade de agenda de todos os grupos. }\end{array}$ & 0,3 & 0,142857143 & 0,042857143 \\
\hline FAZ2 & $\begin{array}{l}\text { Acessível para todos } \\
\text { os membros }\end{array}$ & $\begin{array}{l}\text { O modelo de análise foi coproduzido e } \\
\text { disponibilizado com todos os grupos de } \\
\text { pesquisa e stakeholders por meio dos } \\
\text { relatórios e compartilhamento de arquivos } \\
\text { nos repositórios on-line }\end{array}$ & 1 & 0,142857143 & 0,142857143 \\
\hline FAZ3 & Qualidade & $\begin{array}{l}\text { O padrão de qualidade deve ser } \\
\text { equivalente aos rigores de pesquisa } \\
\text { científica }\end{array}$ & 1 & 0,142857143 & 0,142857143 \\
\hline FAZ4 & Segurança & $\begin{array}{l}\text { O item segurança foi discutido com os } \\
\text { membros }\end{array}$ & 0,6 & 0,142857143 & 0,085714286 \\
\hline FAZ5 & Usabilidade & $\begin{array}{l}\text { A facilidade de uso do bem comum foi } \\
\text { sendo construída ao longo do projeto. } \\
\text { Contudo restam dúvidas entre parte dos } \\
\text { pesquisadores sobre o desdobramento do } \\
\text { modelo com relação às suas pesquisas } \\
\text { individuais }\end{array}$ & 0,4 & 0,142857143 & 0,057142857 \\
\hline FAZ6 & $\begin{array}{l}\text { Conjunto de tópicos } \\
\text { de campo }\end{array}$ & $\begin{array}{l}\text { Foi definido no decorrer do projeto, por } \\
\text { meio de uma taxonomia }\end{array}$ & 0,7 & 0,142857143 & 0,1 \\
\hline FAZ7 & Semântica & $\begin{array}{l}\text { Foi desenvolvida uma taxonomia a partir } \\
\text { do modelo, em formato SKOS, ou seja, } \\
\text { condizente com padrões da Web Semântica }\end{array}$ & 0,8 & 0,142857143 & 0,114285714 \\
\hline FM & Campo unificador & O Tópico é Fatores humanos & 1 & 1 & 1 \\
\hline
\end{tabular}

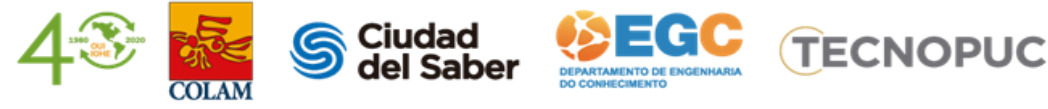




\begin{tabular}{|l|l|l|l|l|l|}
\hline Simb. & Componentes & \multicolumn{1}{|c|}{ Observações } & Valor & Peso & Total \\
\hline TM1 & Lista de tarefas & $\begin{array}{l}\text { O Grupo A passou a utilizar a ferramenta } \\
\text { Trello para gerenciar as atividades dos } \\
\text { membros. Contudo os demais grupos de } \\
\text { pesquisa não tinham acesso a essas } \\
\text { atividades }\end{array}$ & 0,5 & 0,5 & 0,25 \\
\hline TM2 & Rede de agentes & $\begin{array}{l}\text { O repositório do projeto conta com uma } \\
\text { lista dos nomes e contatos de alguns } \\
\text { membros. O ideal é que todos os membros } \\
\text { tivessem acesso a todos os contatos dos } \\
\text { integrantes da comunidade }\end{array}$ & 0,5 & 0,5 & 0,25 \\
\hline TMh & Estrutura de agentes & Não estavam totalmente claras & 0,5 & 1 & 0,5 \\
\hline TMf & Funções dos agentes & Não estavam totalmente claras & 0,5 & 1 & 0,5 \\
\hline HRcp & Código & $\begin{array}{l}\text { Possui um contrato firmado, equivalente ao } \\
\text { código Hash, portanto foi utilizada a média } \\
\text { dos demais componentes deste conjunto de } \\
\text { dados }\end{array}$ & 0,65 & 0,65 \\
\hline
\end{tabular}

Fonte: elaborado pelos autores.

O Conjunto de dados Reference_Policy, apresentado no Quadro 3, descreve as análises qualitativas referentes às políticas simbólicas existentes no ambiente de coprodução do Grupo A, conforme orientações e adaptações do metamodelo de Schneider (2019). Os valores descritos foram estabelecidos por meio de análises documentais e de entrevistas com os integrantes do Grupo A.

Quadro 3- Conjunto de dados Reference_Policy (Política de referência)

\begin{tabular}{|l|l|l|r|r|r|}
\hline \multicolumn{1}{|c|}{ Simb. } & \multicolumn{1}{|c|}{ Componentes } & \multicolumn{1}{|c|}{ Observações } & Valor & Peso & Total \\
\hline RN & Norma referencial & $\begin{array}{l}\text { Entregar os relatórios a cada 4 meses e produzir resultados } \\
\text { concretos das pesquisas semanalmente }\end{array}$ & 1 & 1 & 1 \\
\hline Dc & Conceito Deôtonico & Obrigatória & 1 & 1 & 1 \\
\hline Add & Destinatários & Qualquer membro da comunidade & 1 & 1 & 1 \\
\hline Act & Ativação & Participar do projeto & 1 & 1 & 1 \\
\hline Exp & Expiração & Data final do projeto & 1 & 1 & 1 \\
\hline Sta & Estado & $\begin{array}{l}\text { Havia o monitoramento pelos entregáveis do projeto, bem } \\
\text { como apresentação dos resultados semanais das pesquisas }\end{array}$ & 1 & 1 & 1 \\
\hline Rew & Recompensa & Pagamento a cada 4 meses & 1 & 1 & 1 \\
\hline Pus & Punição & Não estava descrito & 0 & 0 & 0 \\
\hline HRn & & $\begin{array}{l}\text { Possui um contrato firmado, equivalente ao código Hash, } \\
\text { portanto foi utilizada a média dos demais componentes deste } \\
\text { conjunto de dados }\end{array}$ & 1 & 1 & 1 \\
\hline
\end{tabular}

Fonte: elaborado pelos autores.

\section{DADOS DO CONTEXTO SITUACIONAL}

O Conjunto de dados Situational_Negociation, apresentado no Quadro 4, descreve as análises qualitativas referentes às interações ocorridas no ambiente de coprodução do Grupo A, conforme orientações e adaptações do metamodelo de Schneider (2019). Os valores descritos 
foram estabelecidos por meio de análises documentais e de entrevistas com os integrantes do Grupo A.

Quadro 4- Conjunto de dados Situational Negociation (Negociação situacional)

\begin{tabular}{|c|c|c|c|c|c|}
\hline Simb. & Componentes & Observações & Valor & Peso & Total \\
\hline G\&S & Informação & $\begin{array}{l}\text { Para cada mensagem enviada há uma resposta, tanto nos } \\
\text { fóruns, quanto nas funcionalidades. }\end{array}$ & 1 & 1 & 1 \\
\hline Inf1 & $\begin{array}{l}\text { Unidades de } \\
\text { informação - UI }\end{array}$ & Relatório quadrimestral & 1 & 0,3333333 & 0,3333333 \\
\hline Inf2 & $\begin{array}{l}\text { Consistência } \\
\text { das UI }\end{array}$ & $\begin{array}{l}\text { As informações são completas e consistentes e estão de } \\
\text { acordo com o propósito da comunidade. Mas nem sempre } \\
\text { são detalhadas o suficiente. }\end{array}$ & 0,7 & 0,3333333 & 0,2333333 \\
\hline Inf3 & Propostas & $\begin{array}{l}\text { Foram propostos bens e serviços ao longo do projeto, } \\
\text { conforme as discussões ocorriam. Contudo, nem sempre } \\
\text { estavam disponíveis todas as proposições dos outros grupos } \\
\text { de pesquisa }\end{array}$ & 0,6 & 0,3333333 & 0,2 \\
\hline Giv1 & Propostas & $\begin{array}{l}\text { Há discussões semanais para tratar das propostas de } \\
\text { desenvolvimento das pesquisas }\end{array}$ & 1 & 0,5 & 0,5 \\
\hline Giv2 & Proposição & $\begin{array}{l}\text { Na maior parte das reuniões eram enviadas mensagens com } \\
\text { proposições de dinâmicas, oficinas e discussões. Outras } \\
\text { mensagens são respondidas prontamente }\end{array}$ & 0,8 & 0,5 & 0,4 \\
\hline $\mathrm{CN} 1$ & $\begin{array}{l}\text { Negociação de } \\
\text { consenso }\end{array}$ & $\begin{array}{l}\text { A negociação de conceitos ocorria com os membros } \\
\text { participantes das reuniões, porém não há um método claro } \\
\text { para se obter consenso }\end{array}$ & 0,3 & 0,25 & 0,075 \\
\hline $\mathrm{CN} 2$ & $\begin{array}{l}\text { Método de } \\
\text { consenso }\end{array}$ & $\begin{array}{l}\text { O método não é claro. As pautas eram colocadas e os } \\
\text { membros discutiam e entravam em um consenso. } \\
\text { Geralmente o coordenador escolhia a proposição, e os } \\
\text { membros escolhiam se aceitavam ou continuavam a } \\
\text { discussão, até todos concordarem. Contudo este método não } \\
\text { foi descrito e acordado previamente. }\end{array}$ & 0,5 & 0,25 & 0,125 \\
\hline $\mathrm{CN} 3$ & $\begin{array}{l}\text { Objetivo de } \\
\text { consenso }\end{array}$ & $\begin{array}{l}\text { Definido em pautas prévias que ficam disponíveis para todos } \\
\text { os membros }\end{array}$ & 0,8 & 0,25 & 0,2 \\
\hline $\mathrm{CN} 4$ & $\begin{array}{l}\text { Grupo de } \\
\text { consenso }\end{array}$ & Todos os membros presentes nas reuniões podiam opinar & 1 & 0,25 & 0,25 \\
\hline
\end{tabular}

Fonte: elaborado pelos autores.

O Conjunto de dados Situational Negociation, apresentado no Quadro 5, descreve as análises qualitativas referentes às interações ocorridas no ambiente de coprodução do grupo responsável pela condução do desenvolvimento do framework de análise da resiliência, doravante denominado Grupo A, conforme orientações e adaptações do metamodelo de Schneider (2019). Os valores descritos foram estabelecidos por meio de análises documentais e de entrevistas com os integrantes do Grupo A.

Quadro 5- Conjunto de dados Situational_Negociation (Negociação situacional)

\begin{tabular}{|l|l|l|r|r|r|}
\hline Simb. & Unidade de valor & \multicolumn{1}{|c|}{ Observações } & Valor & Peso & Total \\
\hline MFAZ1 & Histórico & $\begin{array}{l}\text { Em todos os relatórios entregues foram detalhadas as } \\
\text { evoluções das pesquisas }\end{array}$ & 1 & 0,3333 & 0,333333333 \\
\hline MFAZ2 & $\begin{array}{l}\text { Produção sobre } \\
\text { Segurança }\end{array}$ & $\begin{array}{l}\text { Não havia membros especificamente tratando do tópico } \\
\text { segurança. Contudo, esse assunto era recorrente nas } \\
\text { reuniões e nos avanços das pesquisas }\end{array}$ & 0,5 & 0,3333 & 0,166666667 \\
\hline
\end{tabular}




\begin{tabular}{|c|c|c|c|c|c|}
\hline Simb. & Unidade de valor & Observações & Valor & Peso & Total \\
\hline MFAZ3 & $\begin{array}{l}\text { Produção sobre } \\
\text { Aprendizado }\end{array}$ & $\begin{array}{l}\text { As reuniões semanais tratavam de compartilhar as notícias e } \\
\text { pesquisas realizadas, além de buscar resolver questões } \\
\text { técnicas }\end{array}$ & 0,7 & 0,3333 & 0,233333333 \\
\hline MTM1 & $\begin{array}{l}\text { Ambiente e grupo } \\
\text { de produção de } \\
\text { conteúdo }\end{array}$ & $\begin{array}{l}\text { A coprodução dos conteúdos foi realizada em workshops, e } \\
\text { membros eram designados para a produção de conteúdo. O } \\
\text { ambiente ainda oferece espaço para melhorias futuras }\end{array}$ & 0,8 & 1 & 0,8 \\
\hline
\end{tabular}

Fonte: elaborado pelos autores.

O último dado do contexto situacional é o dado Situational_Norms, apresentado no Quadro 6, que busca avaliar a cobertura dos elementos mapeados, em relação aos metadados do metamodelo de Schneider (2019). O valor descrito é calculado por x / y, em que x é a quantidade de elementos mapeados na coprodução dos grupos de pesquisa, e y é a quantidade de metadados propostos no metamodelo de Schneider (2019), ou seja, y é igual a 59.

Quadro 6- Dado Situational_Norms (Normas situacionais)

\begin{tabular}{|l|l|r|r|r|}
\hline Simb. & \multicolumn{1}{|c|}{ Observações } & Valor & Peso & Total \\
\hline SNC & $\begin{array}{l}\text { Contagem de elementos instanciados nas estruturas dos contextos } \\
\text { referencial e situacional }\end{array}$ & 0,93220339 & 1 & 0,932203 \\
\hline
\end{tabular}

Fonte: elaborado pelos autores.

A próxima seção apresenta os dados e a aplicação das métricas $\mathrm{R}^{2} \mathrm{SoUP}$.

\section{CONTEXTO DE REPRESENTAÇÃO NO ÂMBITO DO PROJETO DE PESQUISA}

O contexto de representação apresenta os dados calculados a partir dos conjuntos de dados dos contextos referencial e situacional bem como, a aplicação das métricas para calcular o índice de coerência contextual. Após a realização de diversas análises, optou-se por aplicar as métricas das dimensões de referencialidade e senso de unidade. No Quadro 7 são apresentados os dados calculados a partir dos Conjuntos de dados Reference_Common_Contract, Reference_Cultural_Perspective e Reference Norms.

Quadro 7- Dados Situational_Norms (Normas situacionais)

\begin{tabular}{|l|l|l|c|}
\hline Simb. & \multicolumn{1}{|c|}{ Descrição } & Componentes & \multicolumn{1}{|c|}{$\begin{array}{c}\text { Visão geral } \\
\text { referencial }\end{array}$} \\
\hline RCC & $\begin{array}{l}\text { Dados sobre as regras de coprodução, em termos de } \\
\text { funcionamento da rede, e formatos de gestão, } \\
\text { comuns a todo o arranjo coprodutivo }\end{array}$ & Contrato Comum Referencial & 0,606410256 \\
\hline RCP & $\begin{array}{l}\text { Dados sobre assuntos tratados na coprodução e } \\
\text { respectivos papeis e hierarquias assumidos nessa } \\
\text { perspectiva cultural }\end{array}$ & Perspectiva cultural de referência & 0,639285714 \\
\hline
\end{tabular}

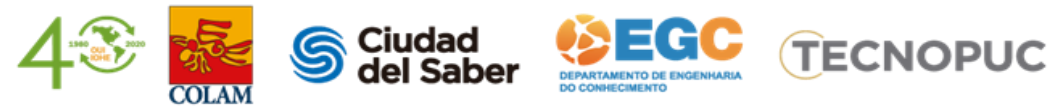




\section{$\mathrm{RN}$}

Dados sobre as restrições de comportamento, sob uma perspectiva cultural, que define como o agente pode ou não agir, nesse contexto de coprodução

Fonte: elaborado pelos autores.

Aplicando-se os valores obtidos no quadro 8, temos os seguintes resultados:

$R f=\frac{R C C+R C P+R P}{3}$

$R f=(0,606410256+0,639285714+0,888888889) / 3 R f=0,711528287$

O diagrama da Figura 4 apresenta os resultados da dimensão referencialidade.

Figura 4 - Diagrama de referencialidade - auto avaliação

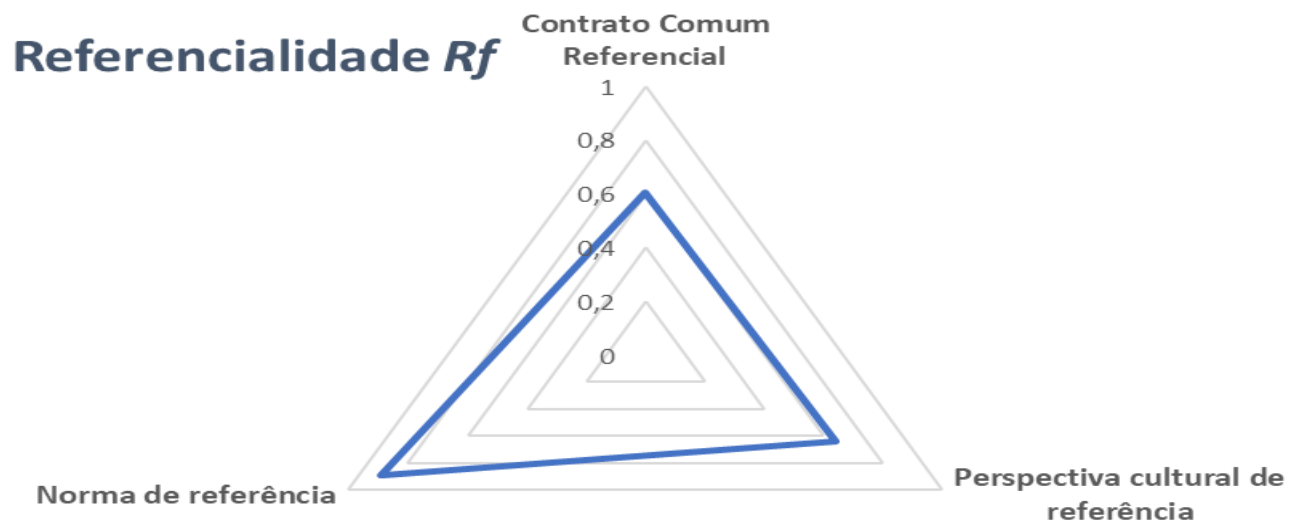

Fonte: elaborado pelos autores

Os dados das variáveis da dimensão Senso de Unidade - SoU, estão descritos no Quadro 9.

Quadro 8- Dado Situational Norms (Normas situacionais)

\begin{tabular}{|l|l|l|}
\hline Fonte & Variável & Dado \\
\hline Produção sobre acessibilidade & MFAZ1 & 1 \\
\hline Histórico & MFAZ2 & 0,5 \\
\hline Produção sobre Segurança & MFAZ3 & 0,7 \\
\hline Ambiente e grupo de produção de conteúdo & MTM1 & 0,8 \\
\hline
\end{tabular}

Fonte: elaborado pelos autores.

Tendo em vista a métrica da dimensão Senso de Unidade SoU de Schneider (2019), foram elaboradas as seguintes adaptações.

$$
\begin{aligned}
& S o U=\frac{(M F A 1+M F A 2+M F A 3+M T M 1)}{4} \\
& S o U=\left(\frac{1+0,5+0,7+0,8)}{4} \quad \text { SoU }=0,75\right.
\end{aligned}
$$

Dessa forma, com a adaptação do índice de Coerência Contextual Cc, apresentado com a equação, em que $R f$ é a dimensão Referencialidade, e $S o U$ é a dimensão Senso de Unidade, temos o seguinte resultado: 


$$
\begin{aligned}
& C c=\frac{\left(R f+S_{o} U\right)}{2} \\
& C_{c}=\frac{(0,711528287+0,75)}{2} \quad C c=0,730764143
\end{aligned}
$$

A próxima seção apresenta as conclusões e discussões deste estudo.

\section{DISCUSSÕES E CONCLUSÕES}

Com a aplicação do metamodelo de Schneider (2019), foi possível realizar um piloto, tendo em vista verificar pontos fortes e fracos sobre a coprodução ocorrida no projeto de pesquisa, no que tange a coprodução em um dos grupos e da ações de coprodução do grupo alvo e os demais grupos do projeto de pesquisa.

Os resultados apresentam algumas oportunidades de melhoria nas interações, tais como, estabelecimento de um método de consenso, ampliação das interações com colaboradores das empresas envolvidas no projeto, bem como a necessidade de ampliação de compartilhamento de conteúdo entre os grupos integrantes do projeto, por meio de um repositório digital que integre as comunicações registradas nas trocas de mensagens.

Além disso, o piloto buscou verificar a viabilidade de aplicação do metamodelo em pesquisas envolvendo os times das empresas envolvidas no estudo. O mapeamento e mensuração das fontes dos símbolos que unem agentes em contextos sociotécnicos de coprodução poderá auxiliar no fortalecimento do potencial de resiliência. Para o projeto, sugerese as seguintes recomendações: a) desenvolver um vocabulário coproduzido com os colaboradores e pesquisadores; b) desenvolver uma taxonomia, a qual pode posicionar os conceitos estabelecidos no projeto em uma estrutura conceitual organizada; e c) estabelecer conceitos que representam os símbolos que unem os agentes na coprodução nas operações das empresas envolvidas no estudo.

Outra possibilidade identificada no estudo foi a de se elaborar narrativas que remetam a resiliência e segurança sob a ótica de fatores humanos, coproduzida por diferentes trabalhadores e especialistas e ilustrada em vídeos. O intuito da elaboração dessas narrativas é iniciar o alinhamento de crenças e valores, com vistas a fortalecer a noção de senso de unidade das 
equipes em torno de uma cultura voltada a resiliência das operações integradas de produção de óleo e gás.

A prova de conceito do sistema de conhecimento, que envolvia pesquisas de referenciais de indicadores, no contexto de operações no setor de óleo e gás, possibilitou a identificação de indicadores candidatos para a incorporação no modelo de conhecimento do projeto. Tal modelo de conhecimento foi materializado em uma taxonomia e foi viabilizado pelas ações de coprodução envolvendo múltiplos atores participes do projeto.

O conhecimento mapeado teve o objetivo de apoiar a análise das funções críticas do sistema e dos seus mecanismos de coordenação que foi realizada pelos pesquisadores. O modelo de conhecimento também foi aplicado na prova de conceito do sistema de conhecimento para suporte às atividades dos pesquisadores. O modelo de conhecimento foi viabilizado por oficinas de coprodução que mobilizaram integrantes dos diferentes grupos de pesquisadores do projeto.

Dentre os pontos que podem ser aprimorados na coordenação e execução de ações de coprodução entre os grupos do projeto analisado, verifica-se a necessidade de se estabelecer uma comunicação mais frequente entre os grupos. Dentre as possibilidades, destaca-se o desenvolvimento de eventos para a discussão das bases epistemológicas de cada grupo participante do projeto, visando o alinhamento conceitual e a promoção da interdisciplinaridade, eventos estes que poderiam auxiliar a minimizar os conflitos existentes em arranjos coprodutivos heterogêneos, como o ambiente das pesquisas do projeto.

A partir dos resultados deste estudo, recomenda-se estabelecer guias de coordenação das ações de coprodução, visando a promoção do compartilhamento, da estruturação e da criação do conhecimento integrando os grupos de pesquisa do projeto. Por exemplo, ações de world café e journal club podem favorecer o compartilhamento das abordagens que cada grupo aplica no plano de pesquisa e favorecer a aproximação entre os membros de cada grupo, ou ainda o compartilhamento de recursos e conhecimentos entre os grupos.

Um método claramente explicitado para se obter o consenso sobre uma proposta coproduzida pelos grupos de pesquisa reforçaria o objetivo comum do projeto. Além disso limites claros estabelecidos sobre o processo de coprodução interdisciplinar, podem apoiar a sinergia dos grupos. Esses são aspectos pontuais de melhoria, sendo que de modo geral, a coprodução realizada no projeto conseguiu alcançar níveis satisfatórios de sinergia entre as 
equipes de pesquisadores e a materialização de artefatos coproduzidos em arranjos interdisciplinares.

\section{AGRADECIMENTOS}

Este trabalho foi realizado com apoio da Agência Nacional de Petróleo, Gás Natural e Biocombustíveis, Brasil (ANP) associado ao investimento de recursos oriundos das Cláusulas de P,D\&I - Regulamento nº 03/2015 (processos: 2016/00187-1 e 2019/00105-3).

\section{REFERENCIAS}

ISO 31000, Risk Management; BS 13500, Organizational Governance; BS 65000, Organizational Resilience; PAS 7000, Supply Chain Risk Management - Supplier Prequalification.

ANSI/ASIS SPC.1-2009 Standard on Organizational Resilience (ASIS, 2009)

Argonne National Laboratory. Petit et al. 2013. Resilience Measurement Index: An Indicator of Critical Infrastructure Resilience.ANL/DIS-13-01. Disponível em $<$ http://www.osti.gov/bridge>. Acesso em: 10 out. 2018.

British Standards Institute 25999 Standard on Business Continuity (BSI, 2010).

Denyer (2017). Organizational Resilience: a summary of academic evidence, business insights and new thinking. BSI and Cranfield School of Management. Disponível em: $<$ https://www.bsigroup.com/organizational-resilience>. Acesso em: 10 out. 2018.

European Agency For Railways. Analysis and Monitoring Initiatives. Disponível em: https://www.era.europa.eu/activities/analysis-and-monitoring_en. Acesso em $10 \mathrm{de}$ janeiro de 2018.

Henriqson et al. (2018). Resiliência em sistemas sociotécnicos complexos: modelo, mensuração e avaliação. WORKING PAPER. Projeto FH1.

Sobrare (2018). Associação Brasileira de Resiliência. Disponível em: <http://sobrare.com.br $>$. Acesso em: 10 out. 2018.

Schneider. V. A coerência dos símbolos que unem agentes em contextos sociotécnicos de coprodução. orientador, Roberto Pacheco,; coorientador, Denilson Sell. Programa de Pós Graduação em Engenharia e Gestão do Conhecimento. Universidade Federal de Santa Catarina - Florianópolis, SC, 2019. 256 p. 